\title{
焦脱镁叶绿酸- $\mathrm{a}$ 的苯腙和氨基酸席夫碱的合成
}

\author{
杨晓英 ${ }^{a}$ 谭广慧 $a, b$ 曲风玉 ${ }^{a, b}$ 金英学 $*, a$ 王进军 $*, c$ \\ $\left({ }^{a}\right.$ 哈尔滨师范大学化学化工学院 哈尔滨 150025) \\ $\left({ }^{b}\right.$ 哈尔滨师范大学生命科学技术学院 哈尔滨 150025) \\ ( 烟台大学化学化工学院 烟台 264005)
}

\begin{abstract}
摘要 以焦脱镁叶绿酸-a 甲酯为原料, 通过 $\mathrm{E}$-环羰基分别与苯肼和氨基酸反应合成了焦脱镁叶绿酸-a 甲酯苯腙和焦脱 镁叶绿酸-a 氨基酸席夫碱. 苯腙的最大吸收波长显著红移. 席夫碱最大吸收波长稍有蓝移, 而其水溶性明显增大. 所有 新化合物的结构经 ${ }^{1} \mathrm{H} N \mathrm{NMR}, \mathrm{MS}$ 或元素分析所证实.
\end{abstract}

关键词＼cjkstart光动力治疗；焦脱镁叶绿酸-a 甲酯(Mppa); 苯腙; 氨基酸; 席夫碱; 水溶性

\section{Synthesis of Phenylhydrazone and Amino acid Schiff Base of Pyropheophorbide-a}

\author{
Yang, Xiaoying ${ }^{a} \quad$ Tan, Guanghui ${ }^{a, b} \quad$ Qu, Fengyu $^{a, b} \quad$ Jin, Yingxue ${ }^{*, a} \quad$ Wang, Jinjun ${ }^{*, c}$ \\ ( ${ }^{a}$ College of Chemistry \& Chemical Engineering, Harbin Normal University, Harbin 150025) \\ ( ${ }^{b}$ College of Life Science and Biotechnology, Haerbin Normal University, Harbin 150025) \\ ( ${ }^{c}$ College of Chemistry and Chemical Engineering, Yantai University, Yantai 264005)
}

\begin{abstract}
Methyl pyropheophorbide-a was used as starting material for the synthesis of phenylhydrazone and amino acid Schiff base by reactions of carbonyl group at $13^{1}$-position. The maximum absorption wavelength of phenylhydrazone caused a red shift. The water-solubility of Schiff base was incremented, and the maximum absorption wavelength shifted hypsochromically. The structures of all these compounds were characterized by ${ }^{1} \mathrm{H}$ NMR, MS and elemental analysis.

Keywords photodynamic therapy (PDT); methyl pyropheophorbide-a (Mppa); phenylhydrazone; amino acid; Schiff base; water-soluble
\end{abstract}

光动力治疗 (photodynamic therapy, PDT) 是正在发 展的新的医疗技术, PDT 在治疗恶性肿瘤和心血管疾病 等领域取得了令人瞩目的成果 ${ }^{[1 \sim 3]}$. PDT 的机理是通过 光敏剂、光照、组织内氧分子发生光动力反应生成活性 氧实现对组织的选择性杀伤 ${ }^{[4,5]}$. PDT 的基础是生物分子 的光敏氧化. 光敏剂接收光激发, 通过一系列反应将能 量传递给生物分子使其氧化, 从而导致细胞丧失生理功 能而调亡. 在此过程中, 光敏剂作为能量载体和桥梁起 着决定性的作用. 因此, 光敏剂是 PDT 的核心物质.

卟啉类是一种具有四吡咯骨架的光敏剂, 许多该类 光敏剂在肿瘤细胞上的选择性地累积能力和较强的荧 光性能, 以及光敏化产生单线态氧的能力, 使其在荧光
诊断和光动力治疗上具有重要的应用 ${ }^{[6 \sim 8]}$. 文献报道了 许多叶绿酸-a 甲酯结构修饰的各种衍生物, 如在 3 位 ${ }^{[9]}$ 、 7 位 ${ }^{[10]} 、 8$ 位 ${ }^{[11]} 、 13$ 位 ${ }^{[12]} 、 17$ 位 ${ }^{[13]} 、 20$ 位 ${ }^{[14]}$ 等上氧化、 卤化和六元环化, 这些化合物在红外-可见光区域有很 强的吸收. 光敏剂的最大吸收波长是考察光敏剂的重要 指标. 目前临床应用的光敏剂吸收波长主要是在红光蓝光区域, 组织穿透深度较浅. 下一代光敏剂的发展趋 势是开发穿透力强的最大吸收波长较大的化合物. 考察 光敏剂的另一个重要指标是影响生物利用度的水脂溶 性. 叶绿酸-a 甲酯的水溶性较差, 修饰结构改善其水脂 溶性是重要的研究内容.

虽然利用 $13^{1}$ 位羰基的反应活性进行结构修饰的报

\footnotetext{
*E-mail: yenghak@hrbnu.edu.cn

Received January 8, 2014; revised February 11, 2014; published online March 10, 2014.

Project supported by the National Natural Sciences of Foundation of China (Nos. 21272048, 21171045), and the Program for Scientific Technological Innovation, Team Construction in Universites of Heilongjiang Province (No. 211TD010).

国家自然科学基金(Nos. 21272048, 21171045)和黑龙江省高效科技创新团队建设计划(No. 2011TD010)资助项目.
} 
道很多 ${ }^{[12]}$, 但与苯肼缩合生成苯腙的反应至今未见报 道. 可以预见, 在二氢卟吩的 $\mathrm{E}$ 环苯腙结构中, 与苯相 连的 $\mathrm{N}$ 原子上的孤对电子所占据未成键杂化轨道与苯 环大 $\pi$ 离域轨道有交叠, 同时也与 $\mathrm{E}-$ 环的 $\mathrm{C}=\mathrm{N}$ 双键 $\pi$ 轨道交叠, 相当于以 $\mathrm{sp}^{3}$ 杂化的氮原子为桥梁, 构建了 二氢卟吩核 $\mathrm{C}=\mathrm{N}-\mathrm{N}$ 苯环离域大 $\pi$ 键, 从而可以拓展 共轭体系, 促使电子激发吸收光谱发生的红移. 本研究 选择了 4 位上不同取代基的六种苯肼与焦脱镁叶绿酸-a 甲酯(Mppa)的 E-环羰基缩合反应, 得到了 6 个苯腙(1), 并且测定了其电子吸收光谱, 结果符合预期设想, 最大 吸收波长 $(\mathrm{Qy})$ 发生显著红移.

羧基亲水性较大，可以大大改善化合物的水溶性. 为了在二氢卟吩外围环上引入羒基基团, 从而提高二氢 卟吩的水溶性, 本研究选择了甘氨酸等 6 种常见的 $\alpha$-氨 基酸, 与焦脱镁叶绿酸-a 甲酯(Mppa)的 E-环羰基缩合反 应, 得到了 6 个氨基酸席夫碱(2), 其水溶性得到明显的 改善(Scheme 1).

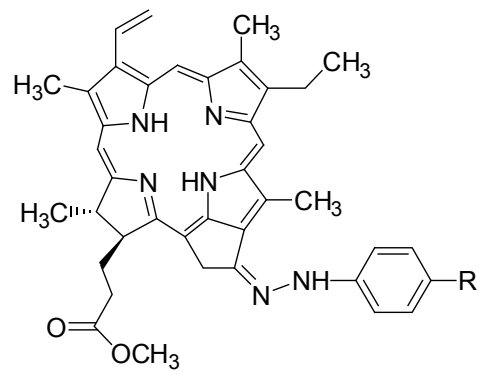

1a $(R=H), 1 b(R=F), 1 c(R=C l)$,

1d $(\mathrm{R}=\mathrm{Br})$, 1e $\left(\mathrm{R}=\mathrm{CH}_{3}\right)$, if $\left(\mathrm{R}=\mathrm{OCH}_{3}\right)$
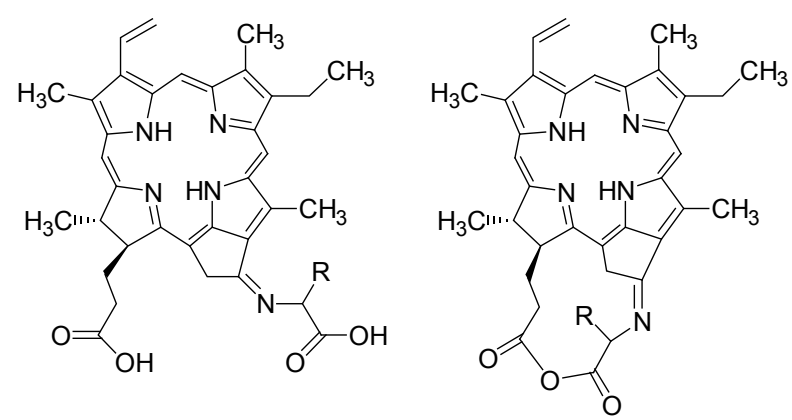

2a $(\mathrm{R}=\mathrm{H}), \mathbf{2 b}\left(\mathrm{R}=\mathrm{CH}_{3}\right), \mathbf{2} \mathbf{c}\left[\mathrm{R}=\mathrm{CH}\left(\mathrm{CH}_{3}\right)_{2}\right], \mathbf{2 d}\left[\mathrm{R}=\mathrm{CH}_{2} \mathrm{CH}\left(\mathrm{CH}_{3}\right)_{2}\right]$, 2e $\left(\mathrm{R}=\mathrm{CH}_{2} \mathrm{SH}\right), \mathbf{2 f}\left[\mathrm{R}=\mathrm{CH}\left(\mathrm{CH}_{3}\right) \mathrm{CH}_{2} \mathrm{CH}_{3}\right]$

\section{Scheme 1}

\section{1 结果与讨论}

\section{1 焦脱镁叶绿酸-a 甲酯(Mppa)的制备和 E-环羰基的 反应}

反应起始物焦脱镁叶绿酸-a 甲酯(Mppa)由叶绿素-a 制备, 具体方法参照文献[15]. 将叶绿素高(主要成分为 叶绿素-a)溶于 $5 \%$ 的硫酸-甲醇溶液中, 在氮气保护下
室温避光摚拌 $24 \mathrm{~h}$. 用布氏漏斗抽滤, 滤液用旋转蒸发 仪浓缩至一半体积后, 加入蒸馏水, 然后用二氯甲烷萃 取两次，有机层用无水硫酸钠干燥后浓缩至干，残余物 用硅胶柱层析分离[洗脱液: $V$ (乙酸乙酯) $: V$ (石油醚 $)=$ $1: 4$ ]得纯脱镁叶绿酸-a 甲酯(Mpa), 产率 7\%. 将所得的 Mpa 溶于冰醋酸中, 加热回流 $6 \mathrm{~h}$, 冷却至室温, 用旋转 蒸发仪除去乙酸, 残余物用二氯甲烷萃取两次, 有机层 用无水硫酸钠干燥后浓缩至干，残余物用硅胶柱层析分 离[洗脱液: $V$ (乙酸乙酯) $: V$ (石油醚) $=1: 4$ ]得纯的焦脱 镁叶绿酸-a 甲酯(Mppa), 产率 $60 \%$. m.p. $214 \sim 215{ }^{\circ} \mathrm{C}$ (Scheme 1).

Mppa 的二氢卟吩大环含有二氮杂 $[18]$ 轮烯的芳香 性碳架结构，E-环羰基类似于苯酮羰基，具有一般羰基 的基本性质，能发生亲核加成反应 ${ }^{[16 ~ 19]}$. E-环羰基由于 与 $[18]$ 轮烯大 $\pi$ 键形成 $\mathrm{p}-\pi$ 共轭, 因而削弱了亲核加成反 应活性，在弱酸溶液中与苯肼发生缩合生成苯腙(1)的 反应时间较长, 在回流条件下需 $5 \mathrm{~h}$ 反应才能结束, 但 产率仍然较好，可以达到 63\% 85\%. E-环羰基在碱性 无水乙醇中，与氨基酸缩合同样需要较长时间，但随着 反应时间的延长, 产物愈加复杂, 17-位脂羧基酯发生水 解，3-位烯键也发生加成水解等反应. 因此反应时间不 宜过长，控制在 $1 \mathrm{~h}$. 焦脱镁叶绿酸-a 氨基酸席夫碱(2) 的产率较低. 焦脱镁叶绿酸-a 甲酯苯腙(1)和焦脱镁叶 绿酸-a 氨基酸席夫碱(2)的结构经核磁共振谱和元素分 析或质谱验证. 化合物 2d 的质谱中发现分子离子峰为 629.3379 , 推断 $13^{1}$ 位氨基酸席夫碱羧基与 17 位羒基间 缩合成环状酸酲. 其它氨基酸席夫碱羧基均末发生成酸 䣶反应. 焦脱镁叶绿酸-a 氨基酸席夫碱(2)的极性与焦 脱镁叶绿酸-a 甲酯相比发生了较大的变化, 在展开剂 苯一丙酮体积比 $5: 1$ 条件下 $R_{\mathrm{f}}$ 值降低 0.5 左右, 说明叶 绿酸-a 氨基酸席夫碱的极性增大, 水溶性增强. 叶绿 酸-a 氨基酸席夫碱的熔点 $>300{ }^{\circ} \mathrm{C}$, 远大于焦脱镁叶绿 素酸-a 甲酯的熔点 $\left(214 \sim 215{ }^{\circ} \mathrm{C}\right)$, 也表明分子间作用力 大, 取向力增强, 分子极性增大.

\subsection{E-环羰基结构转换与光谱变化}

焦脱镁叶绿酸-a 甲酯的 $\mathrm{N}^{21}-\mathrm{N}^{23}$ 的轴向官能团与考 量光敏剂的药效关系密切. A-B 环端向和 C-D 环端向官 能团的性质不但改变其最大吸收波长( $(\mathrm{Qy})$, 也决定分子 的水脂溶性. 特别是 $\mathrm{E}$ 环结构的改变对卟吩母环的理化 性质产生深刻影响. 叶绿素二氢卟吩的光谱性质取决于 大环 $\pi$-电子体系中 HOMO 与 LUMO 的能级差. E-环羰 基成腙后吸收谱带均产生红移. 因为与苯环相连的 $\mathrm{N}$ 原 子是 $\mathrm{sp}^{3}$ 杂化的, $\mathrm{N}$ 上的孤对电子占据未成键的 $\mathrm{sp}^{3}$ 杂化 轨道, 与苯环大 $\pi$ 离域轨道有交叠, 同时也与 $\mathrm{E}$-环的 $\mathrm{C}=\mathrm{N}$ 双键 $\pi$ 轨道交叠, 如 Scheme 2 中 $\mathrm{a}$ 结构所示. 相 


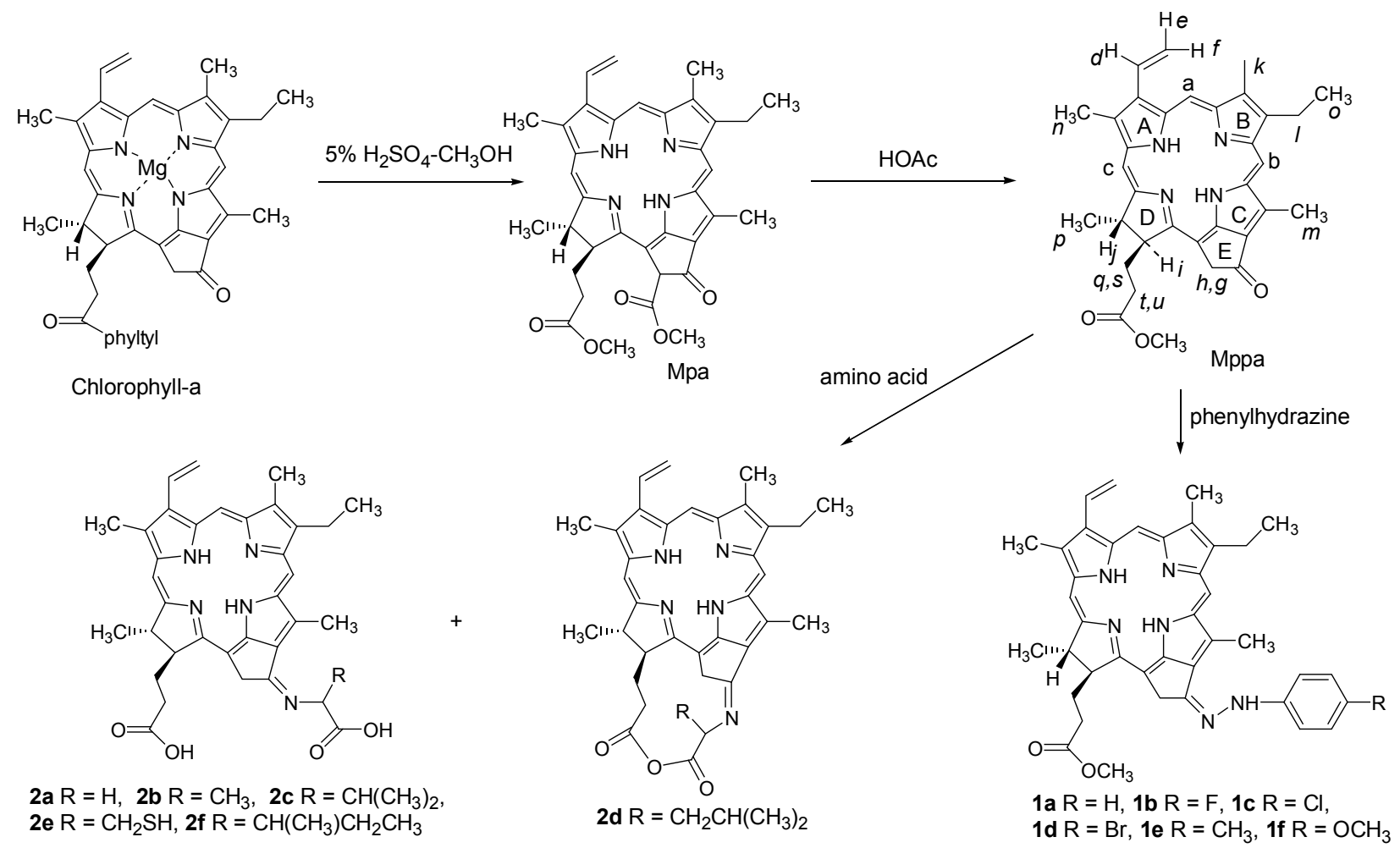

Scheme 2

当于以 $\mathrm{sp}^{3}$ 杂化的氮原子为桥梁, 构建了二氢卟吩核 $\mathrm{C}=\mathrm{N}$ - $\mathrm{N}$ 一苯环离域大 $\pi$ 键，拓展了共轭体系，降低了 HOMO 与 LUMO 的能级差, 促使电子激发吸收光谱发 生较大的红移, 最大红移幅度近 $20 \mathrm{~nm}$. 苯环上取代基 的性质也影响电子激发发射光谱的红移. $\mathrm{R}$ 为拉电子的 $\mathrm{F}, \mathrm{Cl}, \mathrm{Br}$ 时, 由于其吸电子诱导效应, 提高了大环 $\pi$ 体 系 HOMO 轨道的能级, 进一步缩小了电子跃迁能级差, 使 1b, 1c, 1d 吸收光谱红移幅度比没有取代基的苯腙 (1a)略大. $\mathrm{R}$ 为推电子的 $\mathrm{CH}_{3}, \mathrm{OCH}_{3}$ 时, 降低了 LUMO

$$
Q y=680.0 \sim 687.5 \mathrm{~nm} \quad Q y=668.0 \mathrm{~nm}
$$

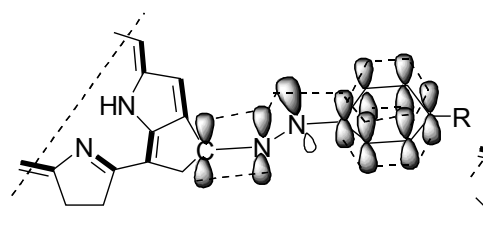

a phenylhydrazone

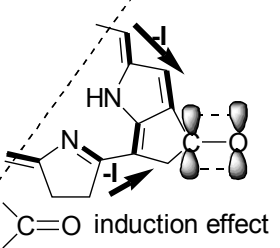

b Mppa
$\mathrm{Qy}=662.0 \sim 668.0 \mathrm{~nm}$

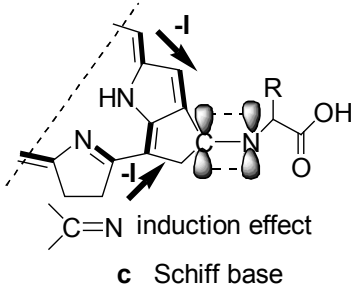

Scheme 2
轨道的能级, 缩小了电子跃迁能级差, 吸收光谱 $\mathrm{Qy}$ 带 发生红移. $\mathrm{OCH}_{3}$ 基的给电子能力较大, 因而 1 f 红移幅 度比 1e大. 1f 同 Mppa 相比 Qy 红移近 $20 \mathrm{~nm}, 1 \mathrm{e}$ 同 Mppa 相比 Qy 红移近 $14.5 \mathrm{~nm}$. 而 E-环羰基成亚胺席夫碱后, 因为氮的电负性小于氧原子, 拉电子能力相对变弱, 二 氢卟吩母核 $[18]$ 轮烯 $\pi$ 电子分散程度减小, 氧化电位降 低, 促使 HOMO 与 LUMO 的能极差变大, 使 $\mathrm{n}-\pi^{*}$ 和 $\pi-\pi^{*}$ 跃迁需要较多的能量, 席夫碱的 Qy 稍有蓝移, 蓝移范 围 $0 \sim 6 \mathrm{~nm}$. 焦脱镁叶绿酸-a 甲酯成席夫碱后 $\mathrm{Qy}$ 在 $662.0 \sim 668.0 \mathrm{~nm}$ 之间.

\section{2 结论}

以叶绿素酸-a 降解产物焦脱镁叶绿酸-a 甲酯为起 始物, 在外接的 E-环羰基部位分别与苯肼和氨基酸发 生缩合反应，合成了焦脱镁叶绿酸-a 甲酯苯腙和焦脱镁 叶绿酸-a 氨基酸席夫碱. 苯腙类衍生物在可见光区吸收 强度较大, 最大吸收波长比焦脱镁叶绿酸-a 甲酯红移 $(\Delta \mathrm{Q}=12 \sim 19.5 \mathrm{~nm})$. 氨基酸席夫碱类衍生物在可见光 区下收强度较大, 最大吸收波长稍有蓝移 $(\Delta \mathrm{Q}=0 \sim 6$ $\mathrm{nm})$, 水溶性显著增大.

\section{3 实验部分}

\section{1 仪器与药品}

Bruker $400 \mathrm{MHz}$ 核磁共振仪( TMS 为内标), $70 \mathrm{eV}$ 
Hitachi VG-7070 质谱仪, UN-160A 紫外分光光度计(波 长单位 nm), Perkin-Elmer 2400 元素分析仪. 叶绿素膏购 自山东广通宝医药有限公司, 其它试剂和药品购自国药 (沈阳)集团. 焦脱镁叶绿酸-a 甲酯的制备方法参考文献 [15]. 所有的反应均在避光氮气保护下进行.

\section{2 脱镁叶绿酸-a 甲酯苯腙(1)的合成}

将 Mppa (30 mg, $0.055 \mathrm{mmol}$ )和 $2 \mathrm{~mL}$ 乙酸溶于 40 $\mathrm{mL}$ 乙醇中, 室温搅拌下滴入肼 $(0.055 \mathrm{mmol})$, 滴毕在氮 气保护下回流 $5 \mathrm{~h}$. 反应结束后冷却至室温, 析出固体, 用布氏漏斗抽滤, 滤饼用少量冷的乙醇洗涤得脱镁叶绿 酸-a 甲酯苯腙粗品, 将粗品用 $10 \mathrm{~mL}$ 乙醇重结晶得精制 的脱镁叶绿酸-a 甲酯苯腙 $(\mathbf{1} \mathbf{a} \sim \mathbf{1 f})$.

1a: 产率 $85 \%$. UV-Vis $\left(\mathrm{CH}_{3} \mathrm{OH}\right) v_{\max }$ (relative intensity): 406.0 (1), 492.5 (0.44), 513.0 (0.33), 622.5 (0.06), $681.5(0.68) \mathrm{nm} ;{ }^{1} \mathrm{H}$ NMR $\left(\mathrm{CDCl}_{3}\right) \delta: 1.77(\mathrm{t}, J=7.6 \mathrm{~Hz}$, $3 \mathrm{H}, \mathrm{Ho}), 1.88$ (d, $J=7.2 \mathrm{~Hz}, 3 \mathrm{H}, \mathrm{Hp}), 2.22 \sim 2.31(\mathrm{~m}, 1 \mathrm{H}$, $\mathrm{Hq}), 2.32 \sim 2.45(\mathrm{~m}, 1 \mathrm{H}, \mathrm{Hu}), 2.54 \sim 2.68(\mathrm{~m}, 1 \mathrm{H}, \mathrm{Hv})$, $2.77 \sim 2.85$ (m,1H, Hs), 3.39 (s, 3H, Hk), 3.54 (s, 3H, Hn), 3.61 (s, 3H, Hm), 3.76 (s, 3H, $\mathrm{OCH}_{3}$ ), 3.85 (q, $J=7.6 \mathrm{~Hz}$, $1 \mathrm{H}, \mathrm{Hl}), 4.43 \sim 4.50$ (m, 1H, Hj), 4.64 (s, 1H, Hi), 5.34 (d, $J=19.2 \mathrm{~Hz}, 1 \mathrm{H}, \mathrm{Hh}), 5.50$ (d, $J=18.0 \mathrm{~Hz}, 1 \mathrm{H}, \mathrm{Hg}), 6.17$ (d, $J=12.8 \mathrm{~Hz}, 1 \mathrm{H}, \mathrm{Hf}), 6.32$ (d, $J=17.6 \mathrm{~Hz}, 1 \mathrm{H}, \mathrm{He})$, 7.88 (s, 1H, PhNH), 8.15 8.23 (m, 1H, Hd), 8.83 (s, 1H, $\mathrm{Hc}), 9.65(\mathrm{~s}, 1 \mathrm{H}, \mathrm{Hb}), 9.79(\mathrm{~s}, 1 \mathrm{H}, \mathrm{Ha})$. Anal. calcd for $\mathrm{C}_{40} \mathrm{H}_{42} \mathrm{~N}_{6} \mathrm{O}_{2}$ : C 75.21, H 6.63, N 13.16; found $\mathrm{C} 75.35, \mathrm{H}$ 6.83, N 13.31 .

1b: 产率 63\%. UV-Vis $\left(\mathrm{CH}_{3} \mathrm{OH}\right) v_{\max }$ (relative intensity): 408.0 (1), 512.0 (0.16), 622.0 (0.02), 682.0 $(0.68) \mathrm{nm} ;{ }^{1} \mathrm{H} \mathrm{NMR}\left(\mathrm{CDCl}_{3}\right) \delta: 1.76(\mathrm{t}, J=7.2 \mathrm{~Hz}, 3 \mathrm{Ho})$, $1.84(\mathrm{~d}, J=7.6 \mathrm{~Hz}, 3 \mathrm{H}, \mathrm{Hp}), 2.21 \sim 2.32(\mathrm{~m}, 1 \mathrm{H}, \mathrm{Hq})$, $2.32 \sim 2.47(\mathrm{~m}, 1 \mathrm{H}, \mathrm{Hu}), 2.50 \sim 2.69(\mathrm{~m}, 1 \mathrm{H}, \mathrm{Hv}), 2.73 \sim$ 2.83 (m, 1H, Hs), 3.31 (s, 3H, Hk), 3.55 (s, 3Hn), 3.60 (s, $3 \mathrm{H}, \mathrm{Hm}), 3.76$ (s, 3H, $\mathrm{OCH}_{3}$ ), 3.83 (q, J=7.6 Hz, 1H, Hl), $4.46 \sim 4.48$ (m, 1H, Hj), 4.64 (s, 1H, Hi), 5.34 (d, $J=19.2$ $\mathrm{Hz}, 1 \mathrm{H}, \mathrm{Hh}), 5.50$ (d, J=18.0 Hz, 1H, Hg), 6.17 (d, $J=$ $12.8 \mathrm{~Hz}, 1 \mathrm{H}, \mathrm{Hf}$ ), 6.32 (d, $J=17.6 \mathrm{~Hz}, 1 \mathrm{H}, \mathrm{He}), 7.83$ (s, $1 \mathrm{H}, \mathrm{PhNH}$ ), 8.16 8.23 (m, 1H, Hd), 8.83 (s, 1Hc), 9.64 (s, $1 \mathrm{H}, \mathrm{Hb}), 9.80$ (s, 1H, Ha). Anal. calcd for $\mathrm{C}_{40} \mathrm{H}_{41} \mathrm{FN}_{6} \mathrm{O}_{2}$ : C 73.15, H 6.29, F 2.89, N 12.80; found C 73.07, H 6.43, F 2.72, N 13.11 .

1c: 产率 70\%. UV-Vis $\left(\mathrm{CH}_{3} \mathrm{OH}\right) v_{\max }$ (relative intensity): 408.0 (1), 511.0 (0.11), 622.0 (0.01), 681.5 (0.54) nm; ${ }^{1} \mathrm{H}$ NMR $\left(\mathrm{CDCl}_{3}\right) \delta: 1.76(\mathrm{t}, J=7.6 \mathrm{~Hz}, 3 \mathrm{Ho})$, $1.85(\mathrm{~d}, J=7.2 \mathrm{~Hz}, 3 \mathrm{H}, \mathrm{Hp}), 2.20 \sim 2.33(\mathrm{~m}, 1 \mathrm{H}, \mathrm{Hq})$, $2.32 \sim 2.45(\mathrm{~m}, 1 \mathrm{H}, \mathrm{Hu}), 2.54 \sim 2.68(\mathrm{~m}, 1 \mathrm{H}, \mathrm{Hv}), 2.77 \sim$
2.85 (m, 1H, Hs), 3.39 (s, 3H, Hk), 3.54 (s, 3H, Hn), 3.61 (s, 3H, Hm), 3.76 (s, 3H, $\left.\mathrm{OCH}_{3}\right), 3.85$ (q, $J=7.6 \mathrm{~Hz}, 1 \mathrm{H}$, Hl), $4.43 \sim 4.50(\mathrm{~m}, 1 \mathrm{H}, \mathrm{Hj}), 4.64(\mathrm{~s}, 1 \mathrm{H}, \mathrm{Hi}), 5.34$ (d, $J=$ $19.2 \mathrm{~Hz}, 1 \mathrm{H}, \mathrm{Hh}), 5.50$ (d, $J=18.0 \mathrm{~Hz}, 1 \mathrm{H}, \mathrm{Hg}), 6.17$ (d, $J=12.8 \mathrm{~Hz}, 1 \mathrm{H}, \mathrm{Hf}), 6.32$ (d, $J=17.6 \mathrm{~Hz}, 1 \mathrm{H}, \mathrm{He}), 7.88$ (s, $1 \mathrm{H}, \mathrm{PhNH}), 8.15 \sim 8.23$ (m, 1H, Hd), 8.83 (s, 1H, Hc), $9.65(\mathrm{~s}, 1 \mathrm{H}, \mathrm{Hb}), 9.79(\mathrm{~s}, 1 \mathrm{H}, \mathrm{Ha})$. Anal. calcd for $\mathrm{C}_{40} \mathrm{H}_{41} \mathrm{ClN}_{6} \mathrm{O}_{2}$ : C 71.36, $\mathrm{H}$ 6.14, $\mathrm{Cl}$ 5.27, N 12.48; found $\mathrm{C}$ 71.51, H 6.33, Cl 5.12, N 12.24.

1d: 产率 $81 \%$. UV-Vis $\left(\mathrm{CH}_{3} \mathrm{OH}\right) \quad v_{\max }$ (relative intensity): 405.5 (1), 512.0 (0.15), 623.0 (0.06), 681.5 (0.73); ${ }^{1} \mathrm{H}$ NMR $\left(\mathrm{CDCl}_{3}\right) \delta: 1.76(\mathrm{t}, J=7.6 \mathrm{~Hz}, 3 \mathrm{H}, \mathrm{Ho})$, $1.88(\mathrm{~d}, J=7.2 \mathrm{~Hz}, 3 \mathrm{H}, \mathrm{Hp}), 2.22 \sim 2.33(\mathrm{~m}, 1 \mathrm{H}, \mathrm{Hq})$, $2.33 \sim 2.46(\mathrm{~m}, 1 \mathrm{H}, \mathrm{Hs}), 2.60 \sim 2.69(\mathrm{~m}, 1 \mathrm{H}, \mathrm{Ht}), 2.74 \sim$ 2.87 (m, 1H, Hu), 3.38 (s, 3H, Hk), 3.53 (s, 3H, Hn), 3.60 (s, 3H, Hm), 3.75 (s, 3H, $\left.\mathrm{OCH}_{3}\right), 3.83$ (q, $J=7.6 \mathrm{~Hz}, 1 \mathrm{H}$, Hl), $4.45 \sim 4.47$ (m, 1H, Hj), 4.64 (s, 1H, Hi), 5.39 (d, $J=$ $18.0 \mathrm{~Hz}, 1 \mathrm{H}, \mathrm{Hh}), 5.50$ (d, $J=18.4 \mathrm{~Hz}, 1 \mathrm{H}, \mathrm{Hg}), 6.18$ (d, $J=10.4 \mathrm{~Hz}, 1 \mathrm{H}, \mathrm{Hf}), 6.34$ (d, $J=18.4 \mathrm{~Hz}, 1 \mathrm{H}, \mathrm{He}), 7.89$ (s, 1H, PhNH), 8.15 8.23 (m, 1H, Hd), 8.83 (s, 1H, Hc), $9.65(\mathrm{~s}, 1 \mathrm{H}, \mathrm{Hb}), 9.79(\mathrm{~s}, 1 \mathrm{H}, \mathrm{Ha})$. Anal. calcd for $\mathrm{C}_{40} \mathrm{H}_{41} \mathrm{BrN}_{6} \mathrm{O}_{2}$ : C 66.94, H 5.76, Br 11.13, N 11.71; found C 67.12, H 5.61, Br 11.29, N 12.01.

1e: 产率 $66 \%$. UV-Vis $\left(\mathrm{CH}_{3} \mathrm{OH}\right) \quad v_{\max }$ (relative intensity): 410.0 (1), 508.0 (0.31), 611.0 (0.21), 682.5 (0.58) nm; ${ }^{1} \mathrm{H}$ NMR $\left(\mathrm{CDCl}_{3}\right) \delta: 1.77(\mathrm{t}, J=7.6 \mathrm{~Hz}, 3 \mathrm{H}$, Ho), 1.88 (d, $J=7.5 \mathrm{~Hz}, 3 \mathrm{H}, \mathrm{Hp}$ ), 2.22 2.32 (m, 1H, Hq), $2.32 \sim 2.45(\mathrm{~m}, 1 \mathrm{H}, \mathrm{Hs}), 2.34\left(\mathrm{~s}, 3 \mathrm{H}, \mathrm{PhCH}_{3}\right), 2.57 \sim 2.68$ $(\mathrm{m}, 1 \mathrm{H}, \mathrm{Ht}), 2.70 \sim 2.83(\mathrm{~m}, 1 \mathrm{H}, \mathrm{Hu}), 3.39$ (s, 3H, Hk), 3.54 (s, 3H, Hn), 3.60 (s, 3H, Hm), 3.75 (s, 3H, $\left.\mathrm{OCH}_{3}\right)$, 3.84 (q, $J=7.6 \mathrm{~Hz}, 1 \mathrm{H}, \mathrm{Hl}), 4.47 \sim 4.48$ (m, 1H, Hj), 4.64 (s, 1H, Hi), 5.35 (d, $J=18.4 \mathrm{~Hz}, 1 \mathrm{H}, \mathrm{Hh}), 5.49$ (d, $J=18.0$ $\mathrm{Hz}, 1 \mathrm{H}, \mathrm{Hg}), 6.18$ (d, J=11.2 Hz, 1H, Hf), 6.34 (d, $J=$ $18.0 \mathrm{~Hz}, 1 \mathrm{H}, \mathrm{He}), 7.83$ (s, 1H, PhNH), 8.19 8.23 (m, 1H, Hd), 8.83 (s, 1H, Hc), 9.63 (s, 1H, Hb), 9.80 (s, 1H, Ha). Anal. calcd for $\mathrm{C}_{41} \mathrm{H}_{4} \mathrm{~N}_{6} \mathrm{O}_{2}$ : C 75.43, $\mathrm{H}$ 6.79, $\mathrm{N}$ 12.87; found C 75.22, H 6.91, N 12.66.

1f: 产率 $70 \%$. UV-Vis $\left(\mathrm{CH}_{3} \mathrm{OH}\right) \quad v_{\max }$ (relative intensity): 410.5 (1), 511.5 (0.04), 628.5 (0.12), 687.5 (0.41) nm; ${ }^{1} \mathrm{H}$ NMR $\left(\mathrm{CDCl}_{3}\right) \delta: 1.77(\mathrm{t}, J=7.6 \mathrm{~Hz}, 3 \mathrm{H}$, Ho), 1.85 (d, J=7.6 Hz, 3H, Hp), $2.20 \sim 2.32$ (m, 1H, Hq), $2.32 \sim 2.44(\mathrm{~m}, 1 \mathrm{H}, \mathrm{Hs}), 2.54 \sim 2.68(\mathrm{~m}, 1 \mathrm{H}, \mathrm{Ht}), 2.74 \sim$ 2.85 (m, 1H, Hu), 3.41 (s, 3H, Hk), 3.54 (s, 3H, Hn), 3.61 (s, 3H, Hm), 3.75 (s, 3H, $\left.\mathrm{OCH}_{3}\right), 3.84$ (q, $J=6.8 \mathrm{~Hz}, 1 \mathrm{H}$, $\mathrm{Hl}), 3.88\left(\mathrm{~s}, 3 \mathrm{H}, \mathrm{PhOCH}_{3}\right), 4.46 \sim 4.48(\mathrm{~m}, 1 \mathrm{H}, \mathrm{Hj}), 4.63 \sim$ 
4.65 (s, 1H, Hi), 5.34 (d, $J=19.2 \mathrm{~Hz}, 1 \mathrm{H}, \mathrm{Hh}), 5.50$ (d, $J=$ $18.0 \mathrm{~Hz}, 1 \mathrm{H}, \mathrm{Hg}), 6.17$ (d, $J=11.6 \mathrm{~Hz}, 1 \mathrm{H}, \mathrm{Hf}), 6.33$ (d, $J=18.0 \mathrm{~Hz}, 1 \mathrm{H}, \mathrm{He}), 7.77$ (s, 1H, $\mathrm{PhNH}), 8.16 \sim 8.23(\mathrm{~m}$, 1H, Hd), 8.83 (s, 1H, Hc), 9.63 (s, 1H, Hb), 9.80 (s, 1H, Ha). Anal. calcd for $\mathrm{C}_{41} \mathrm{H}_{4} \mathrm{~N}_{6} \mathrm{O}_{3}$ : C 73.63, H 6.63, N 12.57; found $\mathrm{C} 73.81, \mathrm{H} 6.32, \mathrm{~N} 12.90$.

\section{3 焦脱镁叶绿酸- $\mathrm{a}$ 氨基酸席夫碱(2)的合成}

将 $\alpha$-氨基酸 $(0.18 \mathrm{mmol})$ 和氢氧化钠粉末 $(0.01 \mathrm{~g}$, $0.25 \mathrm{mmol}$ )溶于 $15 \mathrm{~mL}$ 甲醇中, 充分搅拌, 加热至回流. 将焦脱镁叶绿酸-a 甲酯 $(50 \mathrm{mg}, 0.09 \mathrm{mmol}$ 溶于 $2 \mathrm{~mL}$ 甲 醇中)滴入上述溶液中, 反应 $1 \mathrm{~h}$. 冷却至室温, 用 2 $\mathrm{mol} / \mathrm{L}$ 盐酸酸化至 $\mathrm{pH}=2$. 浓缩除去甲醇溶剂, 残余物 用硅胶柱层析分离 [洗脱剂: $V$ (苯) $: V$ (丙酥 $)=5: 1$ ]得焦 脱镁叶绿酸-a 甲酯氨基酸西夫碱.

2a: 产率 $32 \%$. UV-Vis $\left(\mathrm{CH}_{3} \mathrm{OH}\right) v_{\max }$ (relative intensity): 408.0 (1), 509.5 (0.25), 540.0 (0.22), 611.0 (0.20), $665.0(0.62) \mathrm{nm} ;{ }^{1} \mathrm{H} \mathrm{NMR}\left(\mathrm{CDCl}_{3}\right) \delta: 1.71(\mathrm{t}, J=7.6 \mathrm{~Hz}$, $3 \mathrm{H}, \mathrm{Ho}), 1.81$ (d, J=7.2 Hz, 3H, Hp), 2.23 2.37 (m, $1 \mathrm{Hq}, 1 \mathrm{H}, \mathrm{Hs}), 2.50 \sim 2.60(\mathrm{~m}, 1 \mathrm{H}, \mathrm{Ht}), 2.73 \sim 2.75(\mathrm{~m}, 1 \mathrm{H}$, $\mathrm{Hu}), 3.25$ (s, 3H, Hn), 3.42 (s, 3H, Hm), 3.69 (s, 3H, Hk), 3.71 (q, $J=7.6 \mathrm{~Hz}, 1 \mathrm{H}, \mathrm{Hl}), 4.04 \sim 4.12$ (m, 1H, Hp), $4.31 \sim 4.32(\mathrm{~m}, 1 \mathrm{H}, \mathrm{Hj}), 4.50 \sim 4.51(\mathrm{~m}, 1 \mathrm{H}, \mathrm{Hi}), 5.12(\mathrm{~d}$, $J=20.0 \mathrm{~Hz}, 1 \mathrm{H}, \mathrm{Hh}), 5.28$ (d, $J=21.6 \mathrm{~Hz}, 1 \mathrm{H}, \mathrm{Hg}), 6.19$ (d, $J=11.6 \mathrm{~Hz}, 1 \mathrm{H}, \mathrm{Hf}), 6.29$ (d, $J=18.4 \mathrm{~Hz}, 1 \mathrm{H}, \mathrm{He})$, $8.01 \sim 8.05(\mathrm{~m}, 1 \mathrm{H}, \mathrm{Hd}), 8.57(\mathrm{~s}, 1 \mathrm{H}, \mathrm{Hc}), 9.40(\mathrm{~s}, 1 \mathrm{H}$, $\mathrm{Hb}), 9.51$ (s, 1H, Ha). HRMS (ESI) calcd for $\mathrm{C}_{35} \mathrm{H}_{37} \mathrm{~N}_{5} \mathrm{O}_{4}$ 591.2846, found 591.2862.

2b: 产率 41\%. UV-Vis $\left(\mathrm{CH}_{3} \mathrm{OH}\right) v_{\max }$ (relative intensity): 415.0 (1), 509.5 (0.08), 540.0 (0.07), 611.0 (0.06), $668.5(0.47) \mathrm{nm} ;{ }^{1} \mathrm{H}$ NMR $\left(\mathrm{CDCl}_{3}\right) \delta: 0.94(\mathrm{~d}, J=$ $6.6 \mathrm{~Hz}, 3 \mathrm{H}, \mathrm{Hw}), 1.72$ (t, $J=7.6 \mathrm{~Hz}, 3 \mathrm{H}, \mathrm{Ho}), 1.81$ (d, $J=$ $7.2 \mathrm{~Hz}, 3 \mathrm{H}, \mathrm{Hq}$ ), $2.26 \sim 2.36(\mathrm{~m}, 1 \mathrm{H}, \mathrm{Hu}), 2.53 \sim 2.59(\mathrm{~m}$, $1 \mathrm{H}, \mathrm{Ht}), 2.73 \sim 2.74(\mathrm{~m}, 1 \mathrm{H}, \mathrm{Hs}), 3.25$ (s, 3H, Hn), 3.41 (s, $3 \mathrm{H}, \mathrm{Hm}$ ), 3.68 (s, 3H, Hk), 3.71 (q, $J=7.6 \mathrm{~Hz}, 1 \mathrm{H}, \mathrm{Hl}$ ), $4.07 \sim 4.13$ (m, 1H, Hp), 4.31 (d, $J=8.8 \mathrm{~Hz}, 1 \mathrm{H}, \mathrm{Hj}), 4.50$ (q, $J=8.0 \mathrm{~Hz}, 1 \mathrm{H}, \mathrm{Hi}), 5.13$ (d, $J=19.6 \mathrm{~Hz}, 1 \mathrm{H}, \mathrm{Hg}), 5.28$ (d, $J=20.4 \mathrm{~Hz}, 1 \mathrm{H}, \mathrm{Hh}), 6.17$ (d, $J=11.6 \mathrm{~Hz}, 1 \mathrm{H}, \mathrm{Hf}$ ), $6.31(\mathrm{~d}, J=18.4 \mathrm{~Hz}, 1 \mathrm{H}, \mathrm{He}), 8.02 \sim 8.05(\mathrm{~m}, 1 \mathrm{H}, \mathrm{Hd})$, 8.56 (s, 1H, Hc), 9.40 (s, 1H, Hb), 9.52 (s, 1H, Ha). HRMS (ESI) calcd for $\mathrm{C}_{36} \mathrm{H}_{39} \mathrm{~N}_{5} \mathrm{O}_{4} \mathrm{Na}[\mathrm{M}+\mathrm{Na}]^{+}$628.2900, found. 628.28841 .

2c: 产率 44\%. UV-Vis $\left(\mathrm{CH}_{3} \mathrm{OH}\right) \quad v_{\max }$ (relative intensity): 415.0 (1), 509.5 (0.02), 540.0 (0.012), 611.0 (0.011), 668.5 (0.43) nm; ${ }^{1} \mathrm{H} \mathrm{NMR}\left(\mathrm{CDCl}_{3}\right) \delta: 0.91$ (d, $J=$ $6.3 \mathrm{~Hz}, 6 \mathrm{H}, \mathrm{Hx}), 0.95 \sim 1.06(\mathrm{~m}, 1 \mathrm{H}, \mathrm{Hw}), 1.12 \sim 1.21(\mathrm{~m}$,
2H, Hw), 1.72 (t, $J=7.6 \mathrm{~Hz}, 3 \mathrm{H}, \mathrm{Ho}), 1.83$ (d, $J=7.2 \mathrm{~Hz}$, $3 \mathrm{H}, \mathrm{Hq}$ ), $2.25 \sim 2.31(\mathrm{~m}, 2 \mathrm{H}, \mathrm{Hu}$ and $\mathrm{Hv}), 2.52 \sim 2.61(\mathrm{~m}$, $1 \mathrm{H}, \mathrm{Ht}), 2.71 \sim 2.82(\mathrm{~m}, 1 \mathrm{H}, \mathrm{Hs}), 3.25$ (s, 3H, Hn), 3.41 (s, $3 \mathrm{H}, \mathrm{Hm}$ ), 3.69 (s, 3H, Hk), 3.71 (q, $J=7.6 \mathrm{~Hz}, 1 \mathrm{H}, \mathrm{Hl}$ ), $4.04 \sim 4.12$ (m, 1H, Hp), $4.31 \sim 4.41(\mathrm{~m}, 1 \mathrm{H}, \mathrm{Hj}), 4.50 \sim$ 4.54 (m, 1H, Hi), 5.10 (d, J=20.0 Hz, 1H, Hh), 5.26 (d, $J=21.6 \mathrm{~Hz}, 1 \mathrm{H}, \mathrm{Hg}), 6.17$ (d, $J=11.6 \mathrm{~Hz}, 1 \mathrm{H}, \mathrm{Hf}), 6.31$ (d, $J=18.4 \mathrm{~Hz}, 1 \mathrm{H}, \mathrm{He}), 8.02 \sim 8.05(\mathrm{~m}, 1 \mathrm{H}, \mathrm{Hd}), 8.56$ (s, 1H, Hc), $9.40(\mathrm{~s}, 1 \mathrm{H}, \mathrm{Hb}), 9.52$ (s, 1H, Ha). HRMS (ESI) calcd for $\mathrm{C}_{38} \mathrm{H}_{43} \mathrm{~N}_{5} \mathrm{O}_{4} 633.3315$, found 633.3325 .

2d: 产率 35\%. UV-Vis $\left(\mathrm{CH}_{3} \mathrm{OH}\right) \quad v_{\max }$ (relative intensity): 415.0 (1), 509.0 (0.02), 540.0 (0.015), 611.0 (0.02), $668.0(0.55) \mathrm{nm} ;{ }^{1} \mathrm{H} \mathrm{NMR}\left(\mathrm{CDCl}_{3}\right) \delta: 0.92(\mathrm{~d}, J=$ $6.4 \mathrm{~Hz}, 6 \mathrm{H}, \mathrm{Hy}), 0.96 \sim 1.13(\mathrm{~m}, 1 \mathrm{H}, \mathrm{Hx}), 1.12 \sim 1.22(\mathrm{~m}$, $2 \mathrm{H}, \mathrm{Hw}), 1.71$ (t, $J=7.2 \mathrm{~Hz}, 3 \mathrm{H}, \mathrm{Ho}), 1.81$ (d, $J=8.4 \mathrm{~Hz}$, $3 \mathrm{H}, \mathrm{Hq}$ ), 2.92 (m, 2H, Hu and $\mathrm{Hv}), 2.54 \sim 2.63(\mathrm{~m}, 1 \mathrm{H}$, $\mathrm{Ht}), 2.62 \sim 2.71(\mathrm{~m}, 1 \mathrm{H}, \mathrm{Hs}), 3.25$ (s, 3H, Hn), 3.41 (s, 3H, $\mathrm{Hm}), 3.68$ (s, 3H, Hk), 3.71 (q, $J=7.2 \mathrm{~Hz}, 1 \mathrm{H}, \mathrm{Hl}), 4.08 \sim$ 4.16 (m, 1H, Hp), 4.30 (d, $J=8.0 \mathrm{~Hz}, 1 \mathrm{H}, \mathrm{Hj}), 4.51$ (q, $J=$ $7.2 \mathrm{~Hz}, 1 \mathrm{H}, \mathrm{Hi}$ ), 5.09 (d, $J=19.6 \mathrm{~Hz}, 1 \mathrm{H}, \mathrm{Hh}), 5.25$ (d, $J=$ $21.8 \mathrm{~Hz}, 1 \mathrm{H}, \mathrm{Hg}), 6.17$ (d, $J=11.2 \mathrm{~Hz}, 1 \mathrm{H}, \mathrm{Hf}), 6.28$ (d, $J=17.6 \mathrm{~Hz}, 1 \mathrm{H}, \mathrm{He}), 8.02$ (q, $J=11.6 \mathrm{~Hz}, 1 \mathrm{H}, \mathrm{Hd}), 8.56$ (s, 1H, Hc), $9.40(\mathrm{~s}, 1 \mathrm{H}, \mathrm{Hb}), 9.52$ (s, 1H, Ha); HRMS (ESI) calcd for $\mathrm{C}_{39} \mathrm{H}_{43} \mathrm{~N}_{5} \mathrm{O}_{3} 629.3366$, found 629.3379 .

2e: 产率 $30 \%$. UV-Vis $\left(\mathrm{CH}_{3} \mathrm{OH}\right) \quad v_{\max }$ (relative intensity): 415.0 (1), 509.5 .0 (0.01), 540.0 (0.01), 611.0 (0.009), 668.0 (0.52) nm; ${ }^{1} \mathrm{H}$ NMR $\left(\mathrm{CDCl}_{3}\right) \delta: 1.71(\mathrm{t}, J=$ $7.6 \mathrm{~Hz}, 3 \mathrm{H}, \mathrm{Ho}), 1.83$ (d, J=7.2 Hz, 3H, Hq), 2.17 (s, 1H, $\mathrm{Hx}), 2.27 \sim 2.33(\mathrm{~m}, 2 \mathrm{H}, \mathrm{Hu}$ and $\mathrm{Hv}), 2.51 \sim 2.56(\mathrm{~m}, 1 \mathrm{H}$, $\mathrm{Ht}), 2.71 \sim 2.74$ (m, 1H, Hs), 3.26 (s, 3H, Hn), 3.41 (s, 3H, $\mathrm{Hm}), 3.68$ (s, 3H, Hk), 3.74 (t, $J=7.6 \mathrm{~Hz}, 1 \mathrm{H}, \mathrm{Hl}), 4.05 \sim$ $4.10(\mathrm{~m}, 1 \mathrm{H}, \mathrm{Hp}), 4.29 \sim 4.31(\mathrm{~m}, 1 \mathrm{H}, \mathrm{Hj}), 4.50 \sim 4.52(\mathrm{~m}$, $1 \mathrm{H}, \mathrm{Hi}), 5.14$ (d, $J=20.0 \mathrm{~Hz}, 1 \mathrm{H}, \mathrm{Hh}), 5.30$ (d, $J=20.0$ $\mathrm{Hz}, 1 \mathrm{H}, \mathrm{Hg}), 6.17$ (d, J=11.6 Hz, 1H, Hf), 6.29 (d, $J=$ $18.0 \mathrm{~Hz}, 1 \mathrm{H}, \mathrm{He}), 8.03(\mathrm{dd}, J=11.6, J=10.6 \mathrm{~Hz}, 1 \mathrm{H}$, Hd), 8.56 (s, 1H, Hc), 9.41 (s, 1H, Hb), 9.53 (s, 1H, Ha). HRMS (ESI) calcd for $\mathrm{C}_{36} \mathrm{H}_{39} \mathrm{~N}_{5} \mathrm{O}_{4} \mathrm{~S}$ 637.2723, found 637.2731

2f: 产率 $27 \%$. UV-Vis $\left(\mathrm{CH}_{3} \mathrm{OH}\right) \quad v_{\max }$ (relative intensity): 415.0 (1), 509.5 (0.017), 540.0 (0.016), 611.0 (0.019), 667.5 (0.51) nm; ${ }^{1} \mathrm{H}$ NMR $\left(\mathrm{CDCl}_{3}\right) \delta: 0.89$ (t, $J=$ $6.8 \mathrm{~Hz}, 6 \mathrm{H}, \mathrm{Hz}), 0.92$ (d, $J=8.3 \mathrm{~Hz}, 3 \mathrm{H}, \mathrm{Hy}), 1.11 \sim 1.18$ (m, 2H, Hx), 1.51 1.54 (m, 1H, Hw), 1.69 (t, $J=7.6 \mathrm{~Hz}$, $3 \mathrm{H}, \mathrm{Ho}), 1.81$ (d, $J=7.6 \mathrm{~Hz}, 3 \mathrm{H}, \mathrm{Hq}$ ), $2.24 \sim 2.29$ (m, 1H, $\mathrm{Hu}), 2.30 \sim 2.36(\mathrm{~m}, 1 \mathrm{H}, \mathrm{Hv}), 2.51 \sim 2.59(\mathrm{~m}, 1 \mathrm{H}, \mathrm{Ht})$, 
$2.66 \sim 2.73(\mathrm{~m}, 1 \mathrm{H}, \mathrm{Hs}), 3.26(\mathrm{~s}, 3 \mathrm{H}, \mathrm{Hn}), 3.41(\mathrm{~s}, 3 \mathrm{H}$, $\mathrm{Hm}), 3.71$ (s, 3H, Hk), 3.72 (q, $J=7.6 \mathrm{~Hz}, 1 \mathrm{H}, \mathrm{Hl}), 4.06 \sim$ 4.10 (m, 1H, Hp), 4.30 (d, $J=8.0 \mathrm{~Hz}, 1 \mathrm{H}, \mathrm{Hj}), 4.51$ (q, $J=$ $7.2 \mathrm{~Hz}, 1 \mathrm{H}, \mathrm{Hi}), 5.09$ (d, $J=11.6 \mathrm{~Hz}, 1 \mathrm{H}, \mathrm{Hh}), 5.25$ (d, $J=$ $21.2 \mathrm{~Hz}, 1 \mathrm{H}, \mathrm{Hg}), 6.19$ (d, J=11.6 Hz, 1H, Hf), 6.27 (d, $J=17.6 \mathrm{~Hz}, 1 \mathrm{H}, \mathrm{He}), 8.03$ (d, $J=11.6 \mathrm{~Hz}, 1 \mathrm{H}, \mathrm{Hd}), 8.56$ (s, 1H, Hc), 9.41 (s, 1H, Hb), 9.52 (s, 1H, Ha). HRMS (ESI) calcd for $\mathrm{C}_{39} \mathrm{H}_{43} \mathrm{~N}_{5} \mathrm{O}_{4}[\mathrm{M}-2 \mathrm{H}]^{+}$645.3315, found 645.3329 .

\section{References}

[1] Betz, U. A. Drug Discovery Today 2005, 10, 1057.

[2] Hopkins, A. L.; Groom, C. R. Nat. Rev. Drug Discovery 2002, 1, 727.

[3] Kossodo, S.; Lamuraglia, G. M. Am. J. Cardiovasc. Drugs 2001, 1, 15.

[4] Dolmans, D. E.; Fukumura, D.; Jain, R. K. Nat. Rev. Cancer 2003, 3,380 .

[5] Gurfinkel, M.; Thompson, A. B.; Ralston, W.; Troya, T. L.; Moore, L.; Moore, T. A.; Gust, J. D.; Tatman, D.; Reynolds, J. S.; Muggenburg, B.; Nilula, K.; Pandey, R.; Mayer, R. H.; Hawrysz, D. J.; Sevickmuraca, E. M. Photochem. Photobiol. 2000, 72, 94.

[6] Zheng, G.; Li, H..; Zhang, M.; Lund, K. S.; Chance, B.; Glickson, J. D. Bioconjugate Chem. 2002, 13, 392.

[7] Zheng, G.; Chen, Yu.; Intes, X.; Chance, B.; Glickson, J. D. J. Porphyrins Phthalocyanines 2004, 8, 1106.

[8] Mikhail, A. G..; Ivan, S. L.; Sergey, V. F.; Alexander, G. T.; Anton, A. S.; Anatoly, A. T.; Alexander, A. K.; Andery, F. M. Mendeleev Commun. 2007, 17, 209.

[9] (a) Tamiaki, H.; Takeuchi, S.; Tsudzuki, S.; Miyatake, T.; Tanikaga, R. Tetrahydron 1988, 54, 6699.

(b) Machida, S.; Isoda, Y.; Kunieda, M.; Tamiaki, H. Tetrahedron Lett. 2012, 53, 6277.

(c) Oba, T.; Uda, Y.; Matsuda, K.; Fukusumi, T.; Satoshi, I.; Hiratani, K.; Tamiaki, H. Bioorg. Med. Chem. Lett. 2011, 21, 2489.

(d) Gryshuk A. L.; Chen, Y. H.; Potter, W.; Ohulchansky, T.; Oseroff, A.; Pandey, R. K. J. Med. Chem. 2006, 49, 1874.

(e) Sasaki, S. I.; Yoshizato, M.; Kunieda, M.; Tamiaki, H. Eur. J. Org. Chem. 2010, 29, 5287.

(f) Liang, B. Y.; Liu, Y.; Xu, X.; Jin, Y. X.; Qi, C. X.; Wang, J. J. Chin. J. Org. Chem. 2013, 33, 2357.

(g) Yin, J. G.; Wang, Z.; Yang, Z.; Jin, Y. X.; Wang, J. J. Chin. J. Org. Chem. 2012, 32, 1936.

[10] (a) Ohkubo, K.; Kotani, H.; Shao, J. G.; Ou, Z. P.; Kadish, K. M.; Li, G. L.; Pandey, R. K.; Fujitsuka, M.; Ito, O.; Imahori, H.; Fukuzumi, S. Angew. Chem., Int. Ed. 2004, 43, 853.

(b) Liu, X.; Sternberg, E.; Dolphin, D. J. Org. Chem. 2008, 73, 6542 .

[11] (a) Li, G.; Graham, A.; Chen, Y. H.; Dobhal, M. P.; Morgan, J.; Zheng, G.; Kozyrev, A.; Oseroff, A.; Dougherty, T. J.; Pandey, R. K. J. Med. Chem. 2003, 46, 5349.

(b) Li, G. L.; Dobhal, M. P.; Graham, A.; Shibata, M.; Zheng, G.; Kozyrev, A.; Pandey, R. K. J. Org. Chem. 2003, 68, 3762. (c) Graham, A.; Li, G. L.; Chen, Y. H.; Morgan, J.; Oseroff1, A.; Dougherty, T. J.; Pandey. R. K. Photochem. Photobiol. 2003, 77, 561 .

[12] (a) Ma, L. F.; Dolphin, D. Tetrahedron Lett. 1995, 36, 7791.

(b) Katterle, M.; Holzwarth, A. R.; Jesorka. A. Eur. J. Org. Chem. 2006, 25, 414.

(c) Kozyrev, A. N.; Thomas J. Dougherty, T. J.; Pandey, R. K. Chem. Commun. 1998, 34, 481.

(d) Liu, X.; Sternberg, E.; Dolphin, D. Chem. Commun. 2004, 40, 852 .

(e) Mironov, A. F.; Grin, M. A.; Dzardanov, D. V.; Golovina, K. V.; Shim, Y. K. Mendeleev Commun. 2001, 11, 205.

(f) Grin, M. A.; Titeev, R. A.; Brittal, D. I.; Olga, V.; Ulybina, O. V.; Tsiprovskiy, A. G.; Berzina, M. Y.; Lobanova, I. A.; Sivaev, I. B.; VBregadze, V. I.; Mironov, A. F. Mendeleev Commun. 2011, 21,84 .

[13] (a) Kosaka, N.; Tamiaki, K. Eur. J. Org. Chem. 2004, 23, 2325.

(b) Chen, Y. H.; Gryshuk, A.; Achilefu, S.; Ohulchansky, T.; Potter, W.; Zhong, T. X.; Morgan, J.; Chance, B.; Prasad, P. N.; Henderson, B. W.; Oseroff, A.; Pandey, R. K. Bioconjugate Chem. $\mathbf{2 0 0 5}, 16,1264$.

(c) Chen, Y. H.; Zheng, X.; Dobhal, M. P.; Gryshuk, A.; Morgan, J.; Dougherty, T. J.; Oseroff, A.; Pandey, R. K. J. Med. Chem. 2005, 48, 3692.

[14] (a) Mettath, S.; Gang Zheng.; Zielinski, T. J.; Shibata, M.; Alderfer, J. L.; Dougherty, T. J.; Pandey, R. K. Tetrahedron Lett. 2000, 41, 6289.

(b) Mettath, S.; Li, G. L.; Srikrishnan, T.; Mehta, R.; Grossman, Z. D.; Dougherty, T. J.; Pandey, R. K. Org. Lett. 1999, 1, 1193.

[15] Wang, J.-J.; Ji, W.-Y.; Han, G.-F.; Wu, X.-R.; Wang, L. M.; Shen, R. J. Acta Chim. Sinica 2004, 62, 302 (in Chinese).

(王进军, 纪业伟, 韩光范, 邬旭冉, 王鲁敏, 沈荣基, 化学学报, 2004, 62, 302.)

[16] Pandey, R. K.; Tsuchida, T.; Constantine, S.; Zheng, G.; Medforth, C.; Kozyrev, A.; Molhammad, A.; Rodgers, M. A. J.; Smith, K. M.; Douherty, T. J. Med. Chem. 1997, 40, 3770.

[17] Pandey, R. K.; Sumlin, A.; Shiau, F.-Y.; Dougherty, T.; Smith, K. M. Bioorg. Med. Chem. Lett. 1992, 2, 491.

[18] Rungta, A.; Zheng, G.; Missert, J. R.; Potter, W. R.; Dougherty, T.; Pandey, R. K. Bioorg. Med. Chem. Lett. 2000, 10, 1463.

[19] (a) Wang, J.-J.; Li, J.-Z.; Li, Y.-W.; Jakus, J.; Shim, Y.-K. J. Porphyrins Phthalocyanines 2010, 14, 859.

(b) Wang, J.-J.; Li, Y.-W.; Li, J.-Z.; Yin, J.-G. Chin. J. Org. Chem. 2006, 26, 1714 ( in Chinese).

(王进军, 李韵伟, 李家柱, 殷军港, 有机化学, 2006, 26, 1714.)

(c) Wang, L.-M.; Wang, Z.; Yang. Z.; Jin, Y.-X.; Wang, J.-J. Chin. J. Org. Chem. 2012, 32, 2154 (in Chinese).

(王鲁敏，王振，杨泽，金英学，王进军，有机化学，2012，32， 2154.)

(d) Wang, J.-J.; Li, F.-G.; Li, Y.-W. Chin. J. Org. Chem. 2011, 31, 68 (in Chinese).

(王进军, 李付国, 李韵伟, 有机化学, 2011, 31, 68.)

(e) Ji, J.-Y.; Xia, S.-W.; Zhao, L.-L.; Li, J.-Z.; Qi, C.-X.; Wang, J.-J. Chin. J. Org. Chem. 2013, 33, 1457 (in Chinese).

(纪建业, 夏尚文, 赵丽丽, 李家柱, 祁彩霞, 王进军, 有机化学, 2013, 33, 1457.) 